\title{
CONFORMAL VECTOR FIELDS AND YAMABE SOLITONS
}

\author{
NASSER BIN TURKI, BANG-YEN CHEN, AND SHARIEF DESHMUKH
}

\begin{abstract}
In this paper, we use less topological restrictions and more geometric and analytic conditions to obtain some sufficient conditions on Yamabe solitons such that their metrics are Yamabe metrics, that is, metrics of constant scalar curvature. More precisely, we use properties of conformal vector fields to find several sufficient conditions on the soliton vector fields of Yamabe solitons under which their metrics are of Yamabe metrics.
\end{abstract}

\section{INTRODUCTION}

R. Hamilton introduced the notion of Yamabe flow (cf. 9]), in which the metric on a Riemannian manifold is deformed by evolving according to

$$
\frac{\partial}{\partial t} g(t)=-R(t) g(t)
$$

where $R(t)$ is the scalar curvature of the metric $g(t)$. Yamabe solitons correspond to self-similar solutions of the Yamabe flow.

In dimension $n=2$ the Yamabe flow is equivalent to the Ricci flow (defined by $\frac{\partial}{\partial t} g(t)=-2 R i c(t)$, where Ric denotes the Ricci tensor). However, in dimension $n>2$ the Yamabe and Ricci flows do not agree, since the first one preserves the conformal class of the metric but the Ricci flow does not in general.

An $n$-dimensional connected Riemannian manifold $(M, g)$ is said to be a Yamabe soliton if it admits a smooth vector field $\xi$ that satisfies

$$
\frac{1}{2} £_{\xi} g=(R-\lambda) g
$$

where $£_{\xi}$ denotes the Lie derivative with respect to the vector field $\xi$ and $\lambda$ is a constant. We call the vector field $\xi$ in the above definition a soliton vector field for $(M, g)$. In the rest of this paper, we denote the Yamabe soliton satisfying (1.2) by $(M, g, \xi, \lambda)$. A Yamabe soliton is said to be gradient Yamabe soliton if the soliton vector field $\xi$ is gradient of a smooth function. Recall that on a Riemannian manifold $(M, g)$, the metric $g$ is said to be a Yamabe metric if the scalar curvature $R$ is a constant.

2000 Mathematics Subject Classification. 53C21, 35C06, 35J62.

Key words and phrases. Yamabe soliton; Yamabe metric; Yamabe flow. 
Yamabe flow and Yamabe solitons have been studied quite extensively (cf. [1]-[3], [5], [10], [1]). In [6], it is shown that the metric of a compact gradient Yamabe soliton is a Yamabe metric and the same result is achieved in [10] giving a shorter proof.

One of the interesting questions in the geometry of Yamabe solitons is to find conditions on soliton vector fields of Yamabe solitons so that their metrics are of constant scalar curvature, that is, Yamabe metrics. There are two options for such a study, such as imposing stronger topological restrictions and less geometric and analytic conditions, or less topological restrictions and more geometric and analytic conditions. In this paper, we study Yamabe solitons via the second option, merely with the topological restriction that is connected. We then prove several new sufficient conditions on the soliton fields of the Yamabe solitons for their metrics to be Yamabe metrics.

\section{Preliminaries}

Recall that a smooth vector field $X$ on a Riemannian manifold $(M, g)$ is said to be a conformal vector field if the local flow of $X$ consists of local conformal transformations of $(M, g)$, which is equivalent to the fact that the vector field $X$ satisfies

$$
\frac{1}{2} £_{\xi} g=\rho g,
$$

where $\rho$ is a smooth function on $M$ called the potential function of $X$. Thus, from the definition of Yamabe soliton $(M, g, \xi, \lambda)$, it follows that the soliton field $\xi$ is a conformal vector field with potential function $R-\lambda$. Let $\eta$ be smooth 1-form dual to the soliton field $\xi$. Then, we define a skew symmetric tensor field $\varphi$ on the Yamabe soliton $(M, g, \xi, \lambda)$ by

$$
\frac{1}{2} d \eta(X, Y)=g(\varphi X, Y), \quad X, Y \in \mathfrak{X}(M),
$$

where $\mathfrak{X}(M)$ is the Lie algebra of smooth vector fields on $M$.

Using Koszul's formula and equations (1.2), (2.1), the covariant derivative of the soliton vector field $\xi$ is given by

$$
\nabla_{X} \xi=(R-\lambda) X+\varphi X, \quad X \in \mathfrak{X}(M) .
$$

Using equation (2.2), a direct computation gives the following expression for Riemannian curvature tensor of the Yamabe soliton $(M, g, \xi, \lambda)$ :

$$
R(X, Y) \xi=X(R) Y-Y(R) X+(\nabla \varphi)(X, Y)-(\nabla \varphi)(Y, X),
$$

where the covariant derivative $(\nabla \varphi)$ is defined by

$$
(\nabla \varphi)(X, Y)=\nabla_{X} \varphi Y-\varphi\left(\nabla_{X} Y\right)
$$

Since the 2-form $\Omega(X, Y)=g(\varphi X, Y)$ is closed, using equation (2.3), a straight forward computation leads to the following

$$
(\nabla \varphi)(X, Y)=R(X, \xi) Y+Y(R) X-g(X, Y) \nabla R, \quad X, Y \in \mathfrak{X}(M),
$$


where $\nabla R$ is the gradient of the scalar curvature $R$.

Let Ric denote the Ricci tensor of the Yamabe soliton $(M, g, \xi, \lambda)$. Then the Ricci operator $Q$ of the Yamabe soliton is defined by

$$
g(Q X, Y)=\operatorname{Ric}(X, Y),
$$

which is a symmetric operator that satisfies

$$
\sum(\nabla Q)\left(e_{i}, e_{i}\right)=\frac{1}{2} \nabla R,
$$

with $\left\{e_{1}, . ., e_{n}\right\}$ being a local orthonormal frame on the Yamabe soliton $(M, g, \xi, \lambda)$.

Using the skew-symmetry of the operator $\varphi$, and equation (2.3), we obtain

$$
\operatorname{Ric}(Y, \xi)=-(n-1) Y(R)-g\left(Y, \sum(\nabla \varphi)\left(e_{i}, e_{i}\right)\right),
$$

which leads to

$$
Q(\xi)=-(n-1) \nabla R-\sum(\nabla \varphi)\left(e_{i}, e_{i}\right) .
$$

Recall that the Laplace operator $\Delta$ acting on smooth vector fields on a Riemannian manifold $(M, g)$ is an operator $\Delta: \mathfrak{X}(M) \rightarrow \mathfrak{X}(M)$ defined by

$$
\Delta X=\sum\left(\nabla_{e_{i}} \nabla_{e_{i}} X-\nabla_{\nabla_{e_{i}}} e_{i} X\right) .
$$

Thus, using equation (2.2), we get the following

$$
\Delta \xi=\nabla R+\sum(\nabla \varphi)\left(e_{i}, e_{i}\right) .
$$

\section{Reducing metrics of Yamabe solitons to Yamabe metrics}

Now, we prove the first result of this paper.

Theorem 3.1. Let $(M, g, \xi, \lambda)$ be an $n$-dimensional connected Yamabe soliton with $n>2$. If the soliton field satisfies $Q(\xi)+\Delta \xi=\mu \xi$ for a positive constant $\mu$ and the scalar curvature $R$ of $M$ satisfies

$$
\lambda \leq R \leq \frac{n(n-1)}{n-2} \mu,
$$

then the metric $g$ is a Yamabe metric.

Proof. Using equations (2.6) and (2.7), we have

$$
Q(\xi)+\Delta \xi=-(n-2) \nabla R,
$$

which gives

$$
\nabla R=-\frac{\mu}{(n-2)} \xi .
$$

Using above equation and equation (2.2), we compute the Hessian operator $A_{R}$ of the scalar curvature $R$ to be

$$
A_{R}(X)=-\frac{\mu(R-\lambda)}{n-2} X-\frac{\mu}{(n-2)} \varphi X, \quad X \in \mathfrak{X}(M),
$$


that is,

$$
A_{R}(X)+\frac{\mu(R-\lambda)}{n-2} X=-\frac{\mu}{(n-2)} \varphi X .
$$

The left-hand-side of above equation is symmetric, while the right-hand-side is skew-symmetric and consequently we have

$$
A_{R}(X)=-\frac{\mu(R-\lambda)}{n-2} X, \quad \varphi X=0, \quad X \in \mathfrak{X}(M) .
$$

Using the outcome $\varphi=0$, in the equation (2.6), we obtain

$$
Q(\xi)=-(n-1) \nabla R .
$$

Taking divergence on both sides of this equation and using equations (2.2), (2.5), with $\varphi=0$, we conclude

$$
(R-\lambda) R+\frac{1}{2} \xi(R)=-(n-1) \Delta R,
$$

where $\Delta R=\operatorname{div}(\nabla \mathrm{R})$. Using equation (3.1), the above equation leads to

$$
(R-\lambda) R-\frac{n-2}{2 \mu}\|\nabla R\|^{2}=-(n-1) \Delta R .
$$

Now, using equation (3.2) in computing $\Delta R$, and inserting it in above equation, we arrive at

$$
(R-\lambda)\left(R-\frac{n(n-1)}{n-2} \mu\right)=\frac{n-2}{2 \mu}\|\nabla R\|^{2} .
$$

Finally, the bounds on scalar curvature together with above equation confirms that the scalar curvature $R$ is a constant.

Theorem 3.2. Let $(M, g, \xi, \lambda)$ be an n-dimensional connected Yamabe soliton with $n>2$. If the soliton field $\xi$ annihilates the Hessian operator $A_{R}$ and satisfies $\Delta \xi=-\mu \xi$ for a positive constant $\mu$ and if the Ricci curvature in the direction of the soliton field is a constant, then the metric $g$ is a Yamabe metric.

Proof. As the Ricci curvature in the direction of $\xi$ is a constant, we have $\operatorname{Ric}(\xi, \xi)=\nu\|\xi\|^{2}$, where $\nu$ is a constant. Using equations (2.6), (2.7) and $\Delta \xi=-\mu \xi$, we get

$$
Q(\xi)=\mu \xi-(n-2) \nabla R
$$

which on taking the inner product with $\xi$ leads to

$$
(\mu-\nu)\|\xi\|^{2}=(n-2) g(\xi, \nabla R) \text {. }
$$

Taking covariant derivative with respect to $X$ in above equation and using equation (2.2) and the fact that $\xi$ annihilates $A_{R}$, we conclude

$$
2(\mu-\nu) g((R-\lambda) X+\varphi X, \xi)=(n-2) g((R-\lambda) X+\varphi X, \nabla R),
$$

that is,

$$
2(\mu-\nu)(R-\lambda) \xi-(n-2) \nabla R=\varphi(2(\mu-\nu)(R-\lambda) \xi-(n-2) \nabla R) .
$$


Taking the inner product with $2(\mu-\nu)(R-\lambda) \xi-(n-2) \nabla R$ in above equation and using the skew-symmetry of $\varphi$, we conclude that

$$
\nabla R=2 \frac{(\mu-\nu)(R-\lambda)}{n-2} \xi=h \xi,
$$

where

$$
h=2 \frac{(\mu-\nu)(R-\lambda)}{n-2} .
$$

Taking covariant derivative with respect to $X$ in the equation (3.3) and using equations (2.2), we get

$$
A_{R} X=2 \frac{(\mu-\nu)}{n-2} X(R) \xi+h(R-\lambda) X+h \varphi X,
$$

which on using the symmetry of operator $A_{R}$ and the skew-symmetry of $\varphi$ and equation (3.3), leads to

$$
\begin{aligned}
2 h \varphi X & =2 \frac{(\mu-\nu)}{n-2}(g(X, \xi) \nabla R-g(X, \nabla R) \xi) \\
& =4\left(\frac{\mu-\nu}{n-2}\right)^{2}(R-\lambda)(g(X, \xi) \xi-g(X, \xi) \xi)=0 .
\end{aligned}
$$

Thus, we have either $h=0$ or $\varphi=0$. If $h=0$, then equation (3.3) implies $R$ is a constant. Therefore, suppose $\varphi=0$, which in view of equation (2.6), gives

$$
Q(\xi)=-(n-1) \nabla R .
$$

Taking the inner product with $\xi$ in above equation implies

$$
\nu\|\xi\|^{2}=-(n-1) \xi(R),
$$

which on using equation (3.3), gives

$$
\nu\|\xi\|^{2}=-(n-1) h\|\xi\|^{2},
$$

that is,

$$
\|\xi\|^{2}\left(2 \frac{(\mu-\nu)(R-\lambda)(n-1)}{n-2}+\nu\right)=0 .
$$

Note that if $\mu=\nu$, then equation (3.3) would imply $R$ is a constant. Also, if $\xi=0$, the equation (1.2) will imply $R=\lambda$, therefore, now suppose $\mu \neq \nu$, then the above equation gives

$$
R=\lambda-\frac{(n-2) \nu}{2(n-1)(\mu-\nu)},
$$

that is, $R$ is a constant.

In the above theorem, we could replace the condition that soliton field $\xi$ annihilates the Hessian operator $A_{R}$ in exchange with condition on Ricci curvature in the direction of $\xi$ being constant is made stronger, namely $\xi$ satisfies $Q(\xi)=\nu \xi$ for a constant $\nu$ and certain bounds on the scalar curvature. We get the following result. 
Proposition 3.1. Let $(M, g, \xi, \lambda)$ be an $n$-dimensional connected Yamabe soliton $n>2$. If the soliton field $\xi$ satisfies $\Delta \xi=-\mu \xi$, and $Q(\xi)=\nu \xi$ for constants $\mu \geq 0$ and $\nu$, and scalar curvature satisfies

$$
\lambda \leq R \leq n(n-1) \mu,
$$

then the metric $g$ is a Yamabe metric.

Proof. Using equations (2.6) and (2.7), we have

$$
Q(\xi)+\Delta \xi=-(n-2) \nabla R
$$

which gives

$$
\nabla R=\frac{1}{n-2}(\mu-\nu) \xi
$$

Taking covariant derivative in above equation with respect to $X$ and using equation (2.2) gives

$$
A_{R} X-\frac{1}{n-2}(\mu-\nu)(R-\lambda) X=\frac{1}{n-2}(\mu-\nu) \varphi X
$$

which has one side symmetric while other skew-symmetric, and therefore yields $\varphi=0$. Then equations (2.6) and (2.7) respectively give

$$
\nu \xi=-(n-1) \nabla R \quad \text { and } \quad \mu \xi=-\nabla R
$$

which lead to

$$
\nu=(n-1) \mu .
$$

Thus, inserting the value of $\nu$ in equation (3.5) and in $Q(\xi)=\nu \xi$ respectively, give

$$
\nabla R=-\mu \xi, \quad Q(\xi)=(n-1) \mu \xi
$$

Note that if $\mu=0$, then by above equation $R$ is a constant. Therefore from now on we will assume $\mu>0$. Taking divergence in the second equation of (3.7) and using equations (2.2) and (2.5) we get

$$
R(R-\lambda)+\frac{1}{2} \xi(R)=n(n-1) \mu(R-\lambda)
$$

and multiplying above equation by $-\mu$ and using first equation in (3.7) yields

$$
\mu(R-\lambda)(n(n-1) \mu-R)+\frac{1}{2}\|\nabla R\|^{2}=0 .
$$

Hence, using bounds on scalar curvature in above equation implies that $R$ is a constant.

Using the motivation of analytic vector fields on complex manifolds whose local flows leave complex structure invariant, the notion of $\varphi$-analytic conformal vector fields was introduced in [7]. According to [7], a $\varphi$-analytic conformal vector field is a conformal vector field with respect whose local flow, the tensor field $\varphi$ appearing in equation (2.2) is invariant. For examples of $\varphi$-analytic conformal vector fields, see 8$]$.

Equations (3.1) and (3.3) in the proofs of Theorems 3.1 and 3.2, and the first equation in (3.7) suggest that soliton fields under given conditions are 
$\varphi$-analytic conformal vector fields (cf. [7]). In the rest of this paper, we will use this property that the soliton fields being $\varphi$-analytic to prove following two results. First, observe that if the soliton field of the Yamabe soliton $(M, g, \xi, \lambda)$ is $\varphi$-analytic, then we have

$$
£_{\xi} \varphi=0,
$$

which in view of equation (2.2) is equivalent to

$$
(\nabla \varphi)(\xi, X)=0, \quad X \in \mathfrak{X}(M) .
$$

It is known that the soliton field $\xi$ is $\varphi$-analytic if and only if $\nabla R=f \xi$ for a smooth function $f$ on $M$ (cf. [7]). Moreover, if $f=c$, a constant, then $\xi$ is called a constant type- $c \varphi$-analytic conformal vector field.

Theorem 3.3. Let $(M, g, \xi, \lambda)$ be an $n$-dimensional connected Yamabe soliton. If the soliton field is a $\varphi$-analytic vector field of constant type-c with $c<0$ and the scalar curvature satisfies

$$
\lambda \leq R \leq-n(n-1) c,
$$

then the metric $g$ is a Yamabe metric.

Proof. Since $\xi$ is $\varphi$-analytic vector field of constant type $c$, we have

$$
\nabla R=c \xi
$$

which in view of equation (2.2), gives

$$
A_{R} X=c(R-\lambda) X+c \varphi X .
$$

Using the symmetry of $A_{R}$ and the skew-symmetry of $\varphi$, we get $\varphi=0$. Now, using equation (2.4), we get

$$
R(X, \xi) Y=g(X, Y) \nabla R-Y(R) X,
$$

which in view of $\nabla R=c \xi$, gives

$$
\operatorname{Ric}(Y, \xi)=-(n-1) c g(Y, \xi) .
$$

Thus,

$$
Q(\xi)=-(n-1) c \xi .
$$

Using equations (2.5) and (2.2), a straight forward computation gives

$$
\operatorname{div}(\mathrm{Q}(\xi))=\mathrm{R}(\mathrm{R}-\lambda)+\frac{1}{2} \xi(\mathrm{R}) \quad \text { and } \quad \operatorname{div} \xi=\mathrm{n}(\mathrm{R}-\lambda) .
$$

Now, taking divergence in equation (3.9) and multiplying by $c$ gives

$$
c R(R-\lambda)+\frac{1}{2}\|\nabla R\|^{2}=-n(n-1) c^{2}(R-\lambda),
$$

that is,

$$
c(R-\lambda)(R+n(n-1) c)+\frac{1}{2}\|\nabla R\|^{2}=0 .
$$

Then the bounds on the scalar curvature prove that $R$ is a constant. 
The following theorem uses the combination of the length of soliton vector field (the speed of the integral curves of the soliton field) and scalar curvature, that is, the speed of the integral curves of soliton field is controlled by the scalar curvature.

Theorem 3.4. Let $(M, g, \xi, \lambda)$ be a connected Yamabe soliton of positive scalar curvature. If the soliton field is $\varphi$-analytic vector field and the length of soliton field satisfies

$$
\|\xi\|=\sqrt{2 R}
$$

then $g$ is a Yamabe metric.

Proof. Using $\|\xi\|^{2}=2 R$ and equation (2.2), we get

$$
\nabla R=(R-\lambda) \xi-\varphi \xi
$$

which on taking the inner product with $\xi$, leads to

$$
\xi(R)=2(R-\lambda) R .
$$

Now, multiplying equation (3.10) by $2 R$ gives

$$
2 R(R-\lambda) \xi-2 R \varphi \xi=\|\xi\|^{2} \nabla R .
$$

Since $\xi$ is $\varphi$-analytic, after using equation (3.5) and equation (2.4), we find $X(R) \xi=g(X, \xi) \nabla R$, which on substitution $X=\xi$ reads

$$
\xi(R) \xi=\|\xi\|^{2} \nabla R .
$$

Inserting this equation in equation (3.12) gives

$$
2 R(R-\lambda) \xi-2 R \varphi \xi=\xi(R) \xi
$$

which in view of equation (3.11) and $R>0$ yields

$$
\varphi \xi=0 \text {. }
$$

Now, $\xi$ being a $\varphi$-analytic vector field, we have $\nabla R=f \xi$ for a smooth function $f$, which gives

$$
A_{R} X=X(f) \xi+f(R-\lambda) X+f \varphi X .
$$

Using the symmetry of $A_{f}$ and the skew-symmetry of $\varphi$ in above equation, we obtain

$$
2 f \varphi X=g(X, \xi) \nabla f-X(f) \xi
$$

which on using $X=\xi$ and equation (3.14) yields $\|\xi\|^{2} \nabla f=\xi(f) \xi$, that is,

$$
\|\xi\|^{2}\|\nabla f\|^{2}=\xi(f)^{2} .
$$

Now, taking a local orthonormal frame $\left\{e_{1}, e_{2}, . . e_{n}\right\}$, the equation (3.15) gives

$$
4 f^{2}\|\varphi\|^{2}=4 f^{2} \sum\left\|\varphi e_{i}\right\|^{2}=2\|\xi\|^{2}\|\nabla f\|^{2}-2 \xi(f)^{2},
$$

which in view of equation (3.16), gives

$$
f^{2}\|\varphi\|^{2}=0
$$


If $f=0$, then $R$ is a constant. Therefore, suppose $\varphi=0$. Then the equation (3.10) takes the form $\nabla R=(R-\lambda) \xi$, taking divergence on both sides of this equation, we get

$$
\Delta R=\xi(R)+n(R-\lambda)^{2} .
$$

Now, using $\varphi=0$ in equation (2.2), it takes the form $\nabla_{X} \xi=(R-\lambda) X$ and using it for finding the divergence of $Q(\xi)$, gives $\operatorname{div} \mathrm{Q}(\xi)=\mathrm{R}(\mathrm{R}-\lambda)+\frac{1}{2} \xi(\mathrm{R})$, which in view of equation (3.11) implies

$$
\operatorname{div} \mathrm{Q}(\xi)=2 \mathrm{R}(\mathrm{R}-\lambda) .
$$

Also, equation (2.6) gives $Q(\xi)=-(n-1) \nabla R$, which on taking divergence both sides and using equation (3.18) implies

$$
\Delta R=-\frac{2}{n-1} R(R-\lambda) .
$$

Above equation together with equations (3.11) and (3.17) gives

$$
n(R-\lambda)\left(\frac{2}{n-1}+R-\lambda\right)=0
$$

and it proves $R$ is a constant

\section{FURTHer RESULtS}

In this section, we analyze the impact on the Yamabe soliton if the soliton field $\xi$ is closed as well as if it is not closed. Recall that by equation (2.2), if the soliton field is closed, then $\varphi=0$ and if it is not closed, then $\varphi \neq 0$. We shall call the operator $\varphi$ the operator associated to soliton field.

Theorem 4.1. Let $(M, g, \xi, \lambda)$ be an $n$-dimensional connected Yamabe soliton. If the soliton field $\xi$ is closed and the scalar curvature $R$ is a constant along the integral curves of $\xi$, then the metric $g$ is a Yamabe metric.

Proof. Let $\xi$ be closed. Then equation (2.2) takes the form

$$
\nabla_{X} \xi=(R-\lambda) X \text {. }
$$

Define $f=\frac{1}{2}\|\xi\|^{2}$, which on using above equation implies the following expression for the gradient $\nabla f=(R-\lambda) \xi$. Thus the Hessian operator $A_{f}$ of the function $f$ is given by

$$
A_{f}(X)=X(R) \xi+(R-\lambda)^{2} X .
$$

Now, taking the inner product in above equation with a vector field $Y$ and using the fact that the Hessian operator is symmetric we arrive at

$$
X(R) g(Y, \xi)=Y(R) g(X, \xi),
$$

which leads to

$$
X(R) \xi=g(X, \xi) \nabla R \text {. }
$$


Taking the inner product in above equation with $\nabla R$ and replacing $X$ by $\xi$ leads to

$$
\|\xi\|^{2}\|\nabla R\|^{2}=\xi(R)^{2} .
$$

As the scalar curvature $R$ is a constant along the integral curves of $\xi$, that is, $\xi(R)=0$, above equation implies that $R$ is a constant.

Now, in contrast to above theorem, we prove the following in case that the soliton field $\xi$ is not closed.

Theorem 4.2. Let $(M, g, \xi, \lambda)$ be an $n$-dimensional connected Yamabe soliton. If the soliton field $\xi$ is not closed and it annihilates the associated operator $\varphi$, then the metric $g$ is a Yamabe metric.

Proof. Suppose that the soliton field $\xi$ is not closed (that is, $\varphi \neq 0$ ) and that it annihilates $\varphi$, that is, $\varphi(\xi)=0$. Then the function $f=\frac{1}{2}\|\xi\|^{2}$ on using equation (2.2), has for its gradient

$$
\nabla f=(R-\lambda) \xi
$$

Using equation (2.2) and above equation, we find the following expression for the Hessian operator $A_{f}$

$$
A_{f}(X)=X(R) \xi+(R-\lambda)^{2} X+(R-\lambda) \varphi X .
$$

Now, as the Hessian operator $A_{f}$ is symmetric and the associated operator $\varphi$ symmetric, the above equation gives

$$
(R-\lambda) \varphi X=\frac{1}{2}(g(X, \xi) \nabla R-X(R) \xi),
$$

which on taking $X=\xi$ leads to

$$
\xi(R) \xi=\|\xi\|^{2} \nabla R .
$$

Taking the inner product in above equation with $\nabla R$ gives

$$
\xi(R)^{2}=\|\xi\|^{2}\|\nabla R\|^{2} .
$$

Also, using equation (4.1) and a local orthonormal frame $\left\{e_{1}, . ., e_{n}\right\}$, we compute

$$
(R-\lambda)^{2}\|\varphi\|^{2}=\sum_{i}\left\|(R-\lambda) \varphi e_{i}\right\|^{2}=\frac{1}{4}\left(2\|\xi\|^{2}\|\nabla R\|^{2}-2 \xi(R)^{2}\right),
$$

which in view of equation (4.2) leads to

$$
(R-\lambda)^{2}\|\varphi\|^{2}=0 .
$$

Since $\xi$ is not closed, that is, $\varphi \neq 0$, we find that $R$ is a constant.

Acknowledgements: This work is supported by King Saud University, Deanship of Scientific Research, College of Science Research Center. 


\section{REFERENCES}

[1] S. Brendle, Convergence of the Yamabe flow in dimension 6 and higher, Invent. Math. 170 (3) (2007) 541-576.

[2] S. Brendle, Convergence of the Yamabe flow for arbitrary initial energy, J. Differential Geom. 69 (2) (2005) 217-278.

[3] A. Burchard, R. J. Mccan, A. Smith, Explicit Yamabe flow of an asymmetric cigar, Methods Appl. Anal. 15 (1) (2008) 65-80.

[4] B.-Y. Chen, Total mean curvature and submanifolds of finite type, 2nd edition. World Scientific, Hackensack, NJ (2015).

[5] B. Chow, The Yamabe flow on locally conformally flat manifolds with positive Ricci curvature, Comm. Pure Appl. Math. 45 (1992) 1003-1014.

[6] P. Daskalopoulos, N. Sesum, The classification of locally conformally flat Yamabe solitons, Advances in Math. 240 (2013) 346-369.

[7] S. Deshmukh, F. Alsolamy, A note on conformal vector fields on a Riemannian manifold. Colloq. Math. 136(1), 65-73 (2014)

[8] S. Deshmukh, N. Turki, A note on $\varphi$-analytic conformal vector fields, Anal. Math. Phy. DOI 10.1007/s13324-017-0190-8

[9] R. S. Hamilton, The Ricci flow on surfaces. Math. Gen. Relativ. (Santa Cruz, CA, 1986), 237-262, Contemp. Math. 71 (1998).

[10] S. Y. Hsu, A note on compact gradient Yamabe solitons, J. Math. Anal. Appl. 388 (2012) 725-726.

[11] L. Ma, L. Cheng, Properties of non-compact Yamabe solitons, Ann. Glob. Anal. Geom. 40 (2011), 379-387.

[12] K. Yano, Integral Formulas in Riemannian Geometry, Marcel Dekker, New York, 1970.

Department of Mathematics, College of science, King Saud University P.O. BoX-2455 RiYadh-11451, SAUdi ARABiA

E-mail address: nassert@ksu.edu.sa

Department of Mathematics, Michigan State University, 619 Red Cedar Road, EAst Lancing, Michigan 48824-1027 U.S.A.

E-mail address: chenb@msu.edu

Department of Mathematics, College of science, King Saud University P.O. BoX-2455 RiYadh-11451, SAUdi Arabia

E-mail address: shariefd@ksu.edu.sa 